\title{
A NONEXISTENCE RESULT FOR DISCRETE SYSTEMS RELATED TO THE REVERSED HARDY-LITTLEWOOD-SOBOLEV INEQUALITY
}

\author{
TING TANG
}

Abstract. In this paper, we study the Euler-Lagrange system related to the extremal sequences of the discrete reversed Hardy-Littlewood-Sobolev inequality. This system comes into play in estimating bounds of the Coulomb energy and is associated with the study of conformal geometry. By estimating the infinite series, we prove that the nonexistence of super-solutions of the Euler-Lagrange system satisfied by the extremal sequences of this discrete reversed HardyLittlewood-Sobolev inequality.

Mathematics subject classification (2010): 26D15, 40B05, 47J20.

Keywords and phrases: Discrete reversed Hardy-Littlewood-Sobolev inequality, Euler-Lagrange equation, nonexistence of positive solution.

\section{REFERENCES}

[1] W. Chen, C. Li, Regularity of solutions for a system of integral equations, Commun. Pure Appl. Anal., 4 (2005), 1-8.

[2] W. Chen, C. LI, B. OU, Qualitative properties of solutions for an integral equation, Discrete Contin. Dyn. Syst., 12 (2005), 347-354.

[3] W. Chen, C. LI, B. OU, Classification of solutions for a system of integral equations, Comm. Partial Differential Equations, 30 (2005), 59-65.

[4] W. Chen, C. LI, B. OU, Classification of solutions for an integral equation, Comm. Pure Appl. Math., 59 (2006), 330-343.

[5] Z. Cheng, C. LI, An extended discrete Hardy-Littlewood-Sobolev inequality, Discrete Contin. Dyn. Syst., 34 (2014), 1951-1959.

[6] J. Dou, M. Zhu, Reversed Hardy-Littlewood-Sobolev inequality, Internat. Math. Res. Notices, (2015), no.19, 9696-9726.

[7] G. Huang, C. LI, X. YIn, Existence of the maximizing pair for the discrete Hardy-LittlewoodSobolev inequality, Discrete Contin. Dyn. Syst., 35 (2015), 935-942.

[8] C. Jin, C. LI, Qualitative analysis of some systems of integral equations, Calc. Var. Partial Differential Equations, 26 (2006), 447-457.

[9] Y. LEI, On the integral systems with negative exponents, Discrete Contin. Dyn. Syst., 35 (2015), 1039-1057.

[10] Y. LEI, C. LI, Sharp criteria of Liouville type for some nonlinear systems, Discrete Contin. Dyn. Syst., 36 (2016), 3277-3315.

[11] Y. LEI, C. LI, C. MA, Asymptotic radial symmetry and growth estimates of positive solutions to weighted Hardy-Littlewood-Sobolev system, Calc. Var. Partial Differential Equations, 45 (2012), 43 61.

[12] C. Li, J. Villavert, An extension of the Hardy-Littlewood-Polya inequality, Acta Math. Scientia, 31(B) (2011), 2285-2288.

[13] Y. LI, Remark on some conformally invariant integral equations: the method of moving spheres, J. Eur. Math. Soc., 6 (2004), 153-180.

[14] Y. Li AND M. ZHU, Uniqueness theorems through the method of moving spheres, Duke Math. J., 80 (1995), 383-417. 
[15] E. LIEB, Sharp constants in the Hardy-Littlewood-Sobolev and related inequalities, Ann. of Math., 118 (1983), 349-374.

[16] Q. NGo, V. NGuYen, Sharp reversed Hardy-Littlewood-Sobolev inequality on $R^{n}$, Israel J. Math., 220 (2017), 1-35.

[17] E. M. SteIn, Singular integrals and differentiability properties of function, Princetion Math. Series, Vol. 30, Princetion University Press, Princetion, NJ, 1970.

[18] S. Sun, Y. LEI, Fast decay estimates for integrable solutions of the Lane-Emden type integral systems involving the Wolff potentials, J. Funct. Anal., 263 (2012), 3857-3882.

[19] J. XU, Z. Cheng, Y. FAng, An extension of discrete weighted Hardy-Littlewood-Sobolev inequality in space dimension one, Sci. Sin. Math., 45 (2015), 129-140.

[20] X. XU, Uniqueness theorem for integral equations and its application, J. Funct. Anal., 247 (2007), 95-109. 